\title{
Diagnostic value of serum soluble triggering expressed receptor on myeloid cells 1 (sTREM-1) in suspected sepsis: a meta-analysis
}

\author{
Wei Chang, Fei Peng, Shan-Shan Meng, Jing-Yuan Xu and Yi Yang ${ }^{*}$ (1)
}

\begin{abstract}
Background: We aim to synthesize the up-to-date studies to investigate the diagnostic value of serum soluble triggering expressed receptor on myeloid cells 1 (sTREM-1) in suspected sepsis.

Results: A total of 19 studies with 2418 patients were finally enrolled in the meta-analysis. The pooled sensitivity was 0.82 ( $95 \%$ Cl 0.73 to 0.89 ), specificity 0.81 ( $95 \%$ Cl 0.75 to 0.86 ), positive likelihood ratio 4.3 ( $95 \%$ Cl 3.02 to 6.12 ), negative likelihood ratio 0.22 ( $95 \%$ Cl 0.24 to 0.35 ), diagnostic odds ratio 20 (95\% Cl 9 to 41) and AuROC 0.88 ( $95 \%$ $\mathrm{Cl} 0.85$ to 0.91 ). The meta-regression analysis revealed that the sample size, reference standard description, prevalence of sepsis in the trials and consecution of patient recruitment might be the source of heterogeneity.

Conclusions: The serum sTREM-1 had a moderate ability in diagnosis in suspected sepsis based on the current studies. However, more large-scale studies were needed to further evaluate the diagnostic accuracy of sTREM-1.

Keywords: Sepsis, Systemic inflammatory response syndrome, SIRS, Diagnosis, Soluble triggering receptor expressed on myeloid cells 1, sTREM-1
\end{abstract}

\section{Background}

Sepsis is defined as life-threatening organ dysfunction caused by a dysregulated host response to infections, which causes high mortality in the intensive care unit (ICU) and is a grave burden to the public health [1] . Early recognition and diagnosis of sepsis in the high-risk patients with suspected infection is essential for the prompt management and empirical antibiotics therapy, which could potentially improve the mortality in septic patients [2].

The utility of biomarkers in the early recognition, risk stratification, antibiotic stewardship and outcome prediction in septic patients has long been applied in the clinical practice [3]. A myriad of molecules has been under investigation in the early discrimination of sepsis, including Creactive protein, procalcitonin, cytokines and surface markers of circulating leukocytes [4], which could be promising biomarkers in the diagnosis of sepsis.

\footnotetext{
* Correspondence: yiyiyang2004@163.com

Department of Critical Care Medicine, Zhongda Hospital, School of Medicine,

Southeast University, Nanjing, China
}

Triggering receptor expressed on myeloid cells 1 (TREM-1), a member of immunoglobulin family predominantly expressed on the neutrophils and monocytes, was first identified by Bouchon A. et al in 2000 [5], which is upregulated in response of bacterial and fungal infections but poorly expressed in non-infectious inflammation $[6,7]$. The soluble form of TREM-1 (sTREM-1) is shed from cell surface and released into body fluids including plasma, pleural effusion, sputum and urine during the process of infections through proteolytic cleavage by metalloproteinases triggered by lipopolysaccharide $[8,9]$.

The elevated sTREM-1 in the body fluids during infection could be measured directly by immunosorbent assays and used as a tool in discriminating infection from non-infectious inflammation, which makes it a promising candidate in the diagnosis of sepsis. A plethora of studies have been conducted to investigate the value of sTREM-1 as an early biomarker in patients with

(C) The Author(s). 2020 Open Access This article is distributed under the terms of the Creative Commons Attribution 4.0 International License (http://creativecommons.org/licenses/by/4.0/), which permits unrestricted use, distribution, and

reproduction in any medium, provided you give appropriate credit to the original author(s) and the source, provide a link to the Creative Commons license, and indicate if changes were made. The Creative Commons Public Domain Dedication waiver (http://creativecommons.org/publicdomain/zero/1.0/) applies to the data made available in this article, unless otherwise stated. 
suspected infections since its discovery [10], however, its diagnostic accuracy remains undetermined.

Previous meta-analyses has evaluate the diagnostic ability of sTREM-1 in suspected infections [11-13], however with the burgeoning clinical studies of sTREM1 as a diagnostic toolkit in suspected sepsis in recent years, we thought it quite necessary to conduct an upto-date meta-analysis to assess the diagnostic value of serum sTREM-1 in patients with suspected sepsis.

\section{Results}

\section{Study selection}

A total of 514 abstracts were recruited from the search, 31 duplicates were excluded and the remaining 483 were left for screen, within which 98 abstracts were not eligible. In the remaining 385 abstracts, full manuscripts were recruited for further assessment, and 366 articles were excluded with reasons. A final of 19 studies were included in this meta-analysis [14-32] (Fig. 1).

\section{Study characteristics}

A total of 2418 patients was finally enrolled in this meta-analysis, with the average sepsis prevalence of $60.3 \%$. Among the 19 studies included, 17 were prospective $[14,15,17,19-32]$ and two were cross-sectional
$[16,18]$; in five studies traumatic or post-operative patients were enrolled exclusively [16, 19, 22, 28, 29]. Pulmonary infection was the leading cause of infection in 14 studies. Two studies focused on pulmonary [32] or abdominal infections [28] exclusively. The cut-off values ranged from $30 \mathrm{pg} / \mathrm{mL}$ to $60 \mathrm{ng} / \mathrm{mL}$, sensitivity from 49 to 98.3\%, specificity from 40 to $91.7 \%$ and AuROC from 0.61 to 0.978 . Three articles were written in Chinese $[17,26,32]$. (Table 1) The quality assessments of the included studies (Additional file 3) were summarized in Fig. 2.

\section{Syntheses of results}

The synthesis of the 19 studies by the bivariate model yielded a pooled sensitivity of 0.82 ( $95 \%$ CI 0.73 to 0.89 ), specificity of 0.81 (95\% CI 0.74 to 0.86 ), PLR of 4.3 (95\% CI 3.0 to 6.1), NLR of 0.22 (95\% CI 0.14 to 0.35 ) and DOR of 20 (95\% CI 9 to 41) (Fig. 3), with AuROC of 0.88 (95\% CI 0.85 to 0.91) (Fig. 4). The proportion of heterogeneity likely due to threshold effect was 0.20 . We assumed the pre-test probability of $60 \%$ as the overall average sepsis prevalence concluded from the trials enrolled and yielded the post-test positive probability of $87 \%$ and negative of $26 \%$, as illustrated in the Fagan's nomogram (Fig. 5). The scattergram indicated that the

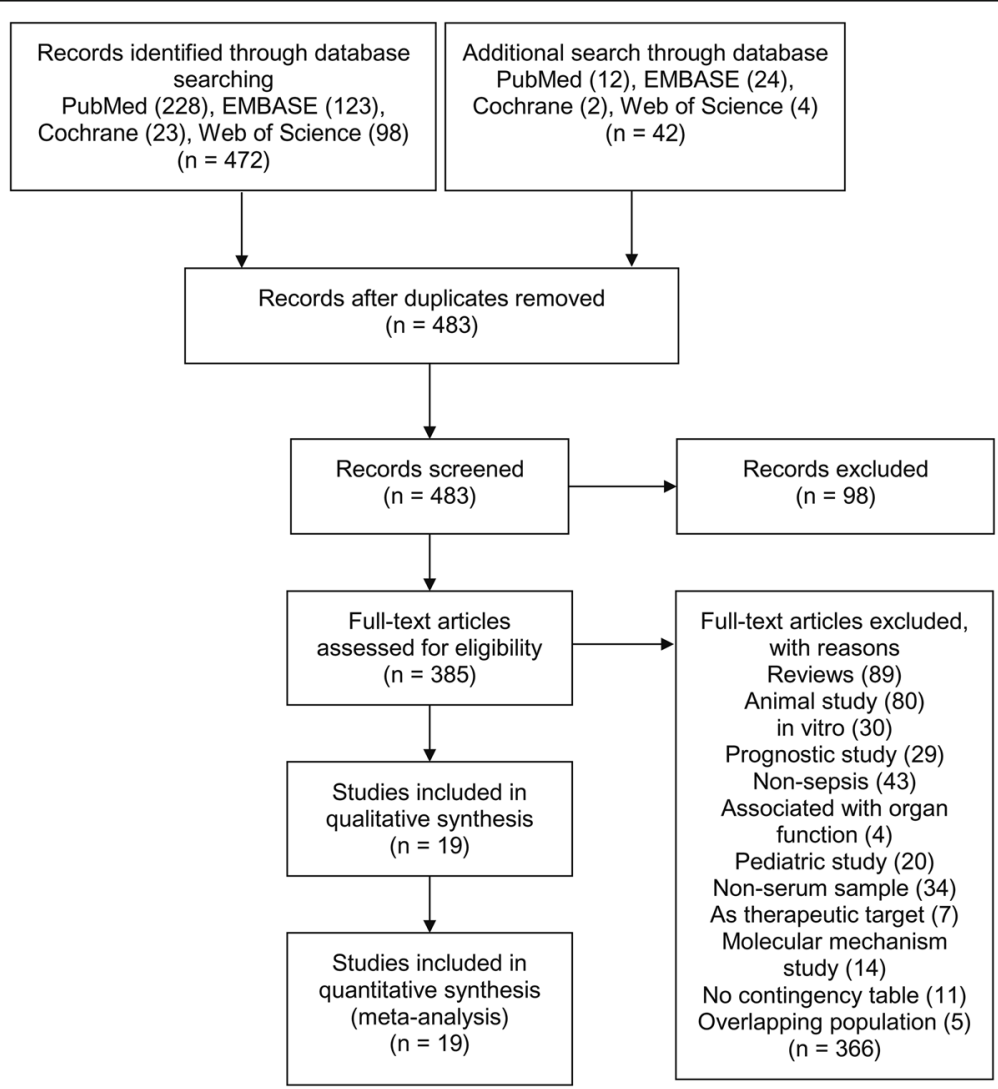

Fig. 1 Flow diagram. Flow chart of study screen and selection 


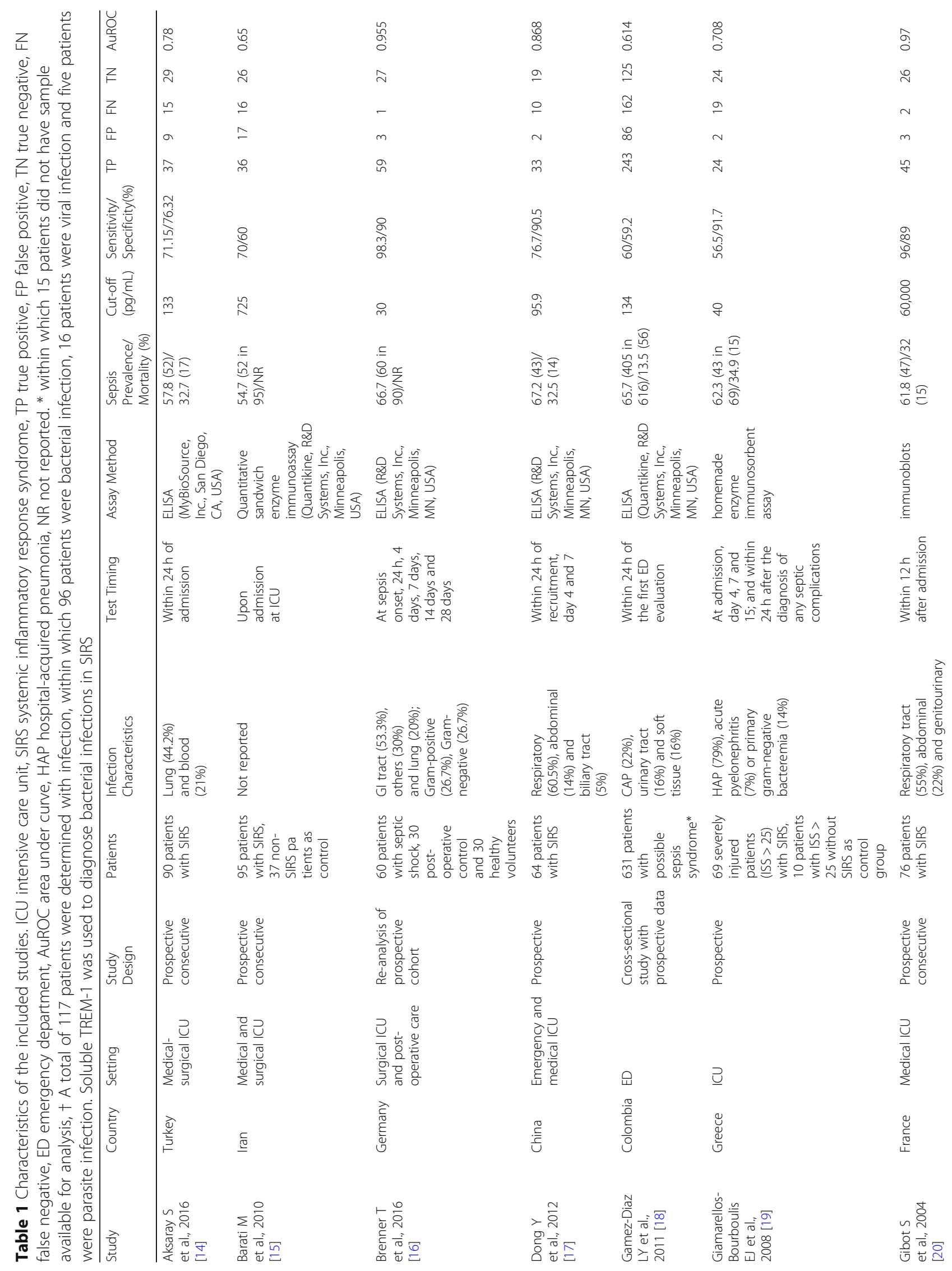




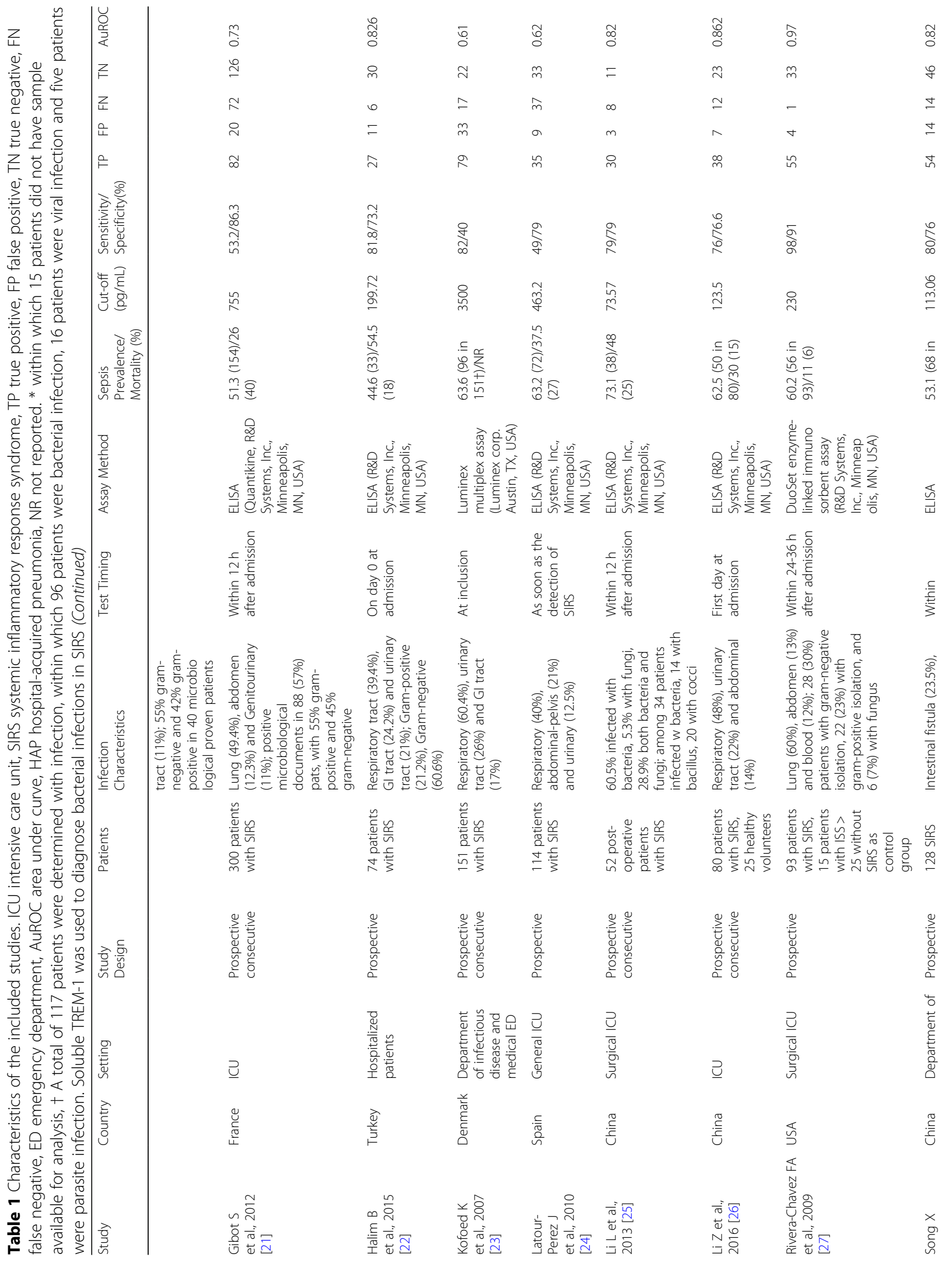




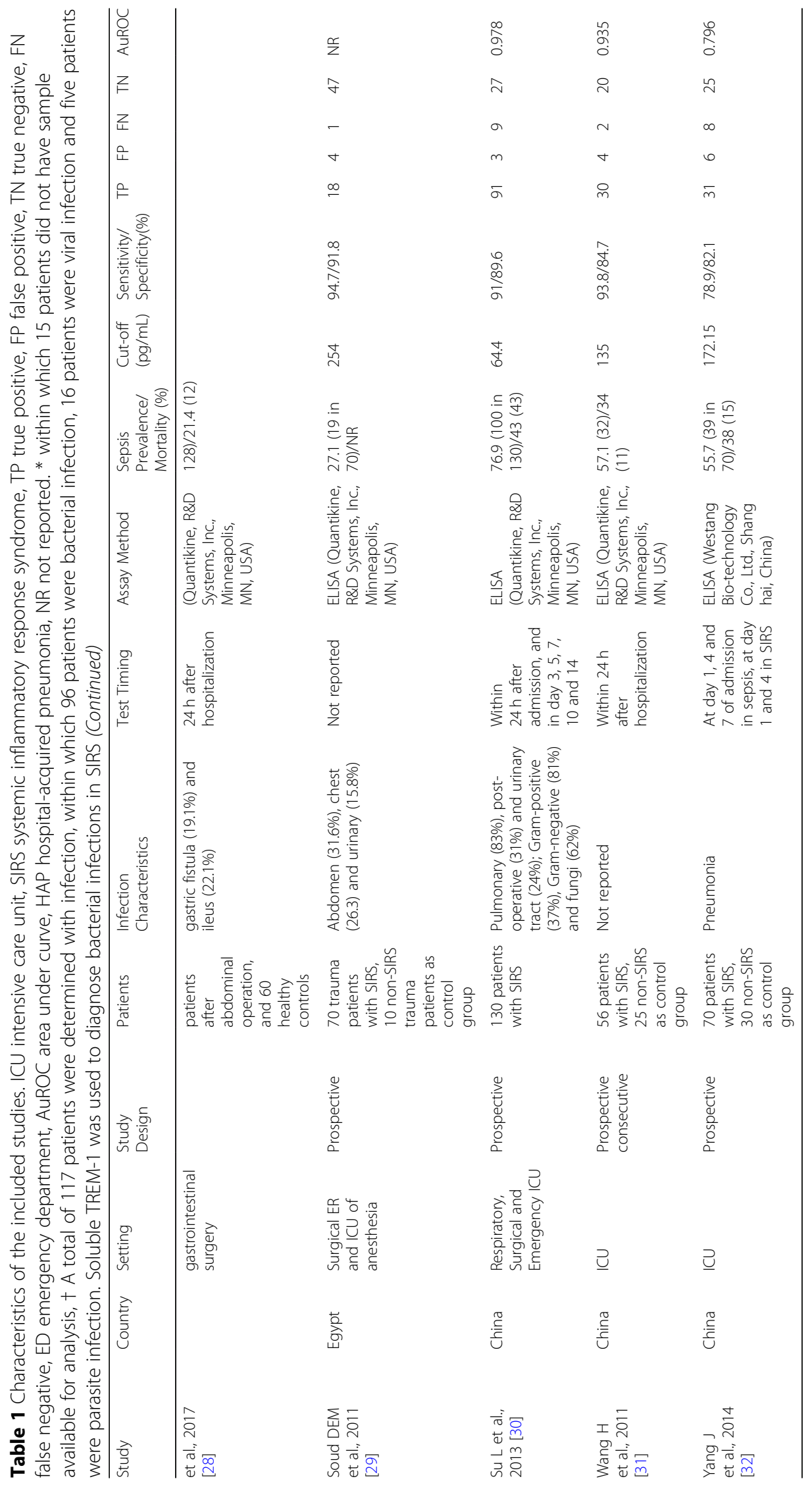




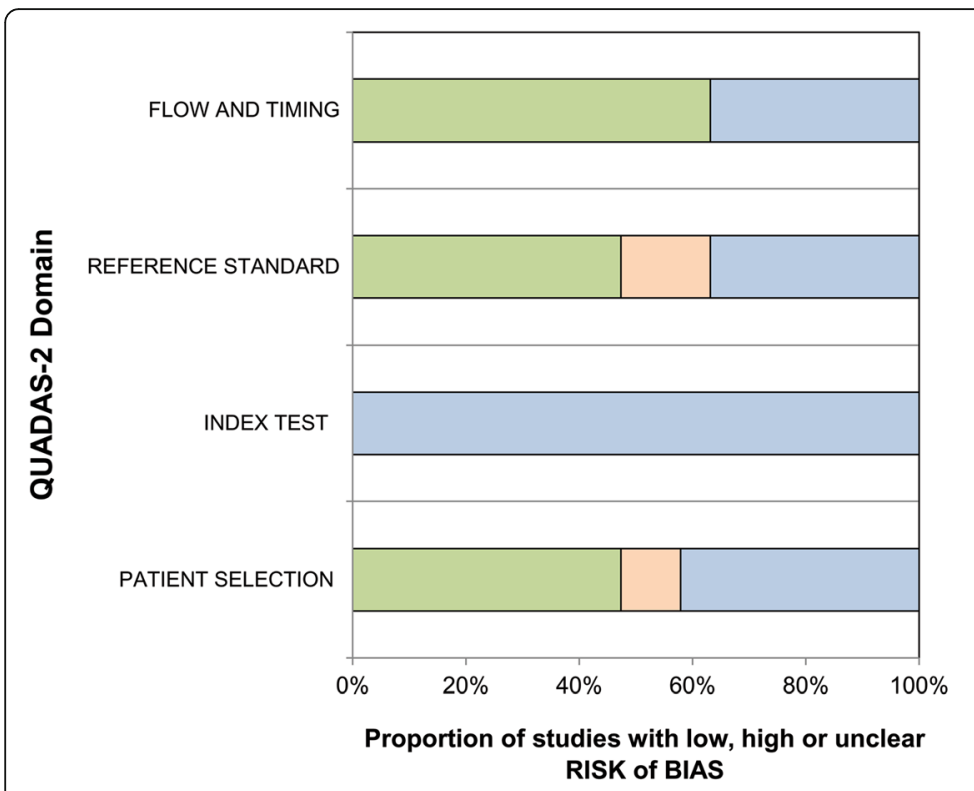

口Low $\square$ High uUnclear

Fig. 2 The qualities of the included studies assessed by QUADAS-2 tool

sTREM-1 of limited clinical diagnostic value with PLR < 10 and NLR $>0.1$ (no exclusion or confirmation) (Fig. 6).

\section{Risk of bias and sub-group analysis}

The studies were divided according to the cut-off values of sTREM-1, and the results revealed the AuROC of 0.87 (95\% CI 0.84 to 0.90 ), sensitivity of 0.81 ( $95 \% 0.73$ to 0.87$)$ and specificity of 0.80 (95\% CI 0.73 to 0.86$)$ at the cut-off range of 30 to $199.72 \mathrm{pg} / \mathrm{mL}$; and AuROC of 0.89 ( $95 \%$ CI 0.86 to 0.91 ), sensitivity of 0.85 (95\% CI 0.64 to 0.95 ) and specificity of 0.80 (95\% CI 0.65 to 0.90$)$ at the cut-off range of $230 \mathrm{pg} / \mathrm{mL}$ to $60 \mathrm{ng} / \mathrm{mL}$ (Additional file 4).

We conducted the meta-analysis in the sub-group of 7 prospective trials conducted in the ICU, in which the patients with SIRS were consecutively recruited $[14,15,20$, $21,25,26,31]$. In this relatively homogenous population of patients, the result revealed that the pooled sensitivity of 0.80 ( $95 \%$ CI 0.68 to 0.89 ), specificity of 0.76 ( $95 \%$ CI 0.64 to 0.84 ), PLR of 3.3 ( $95 \%$ CI 2.0 to 5.5 ), NLR of 0.26 (95\% 0.14 to 0.48 ) and DOR of 13 (95\% CI 4 to 38 ) (Additional file 5: Figure S1).

A categorical univariate meta-regression analysis was conducted including the factors which could potentially bring bias to the results. We found that the sample size, and the reference standard description was possibly related with the heterogeneity in both sensitivity and specificity, and the prevalence of sepsis and the consecution of patient recruitment was possibly related with the heterogeneity in specificity (Fig. 7). Sub-group analyses were conducted according to the factors possibly related to the heterogeneity. (Additional file 6: Figure S2).
The Deek's funnel plot was constructed and potential bias could be inspected with the $P$ value of 0.002 , suggesting publication bias should be considered (Fig. 8).

\section{Disscussion}

In this study, we synthesized 19 trials and found that the sTREM-1 has a moderately accuracy in diagnosis of sepsis in high-risk patients with the pooled sensitivity of sensitivity of 0.82 (95\% CI 0.73 to 0.89 ), specificity of 0.81 (95\% CI 0.74 to 0.86 ) and the AuROC of 0.88 (95\% CI 0.85 to 0.91$)$.

In previous meta-analysis by Wu et al including 11 trials of 1795 patients, they found the pooled sensitivity and specificity of 0.79 (95\% CI 0.65 to 0.89 ) and 0.80 (95\% CI 0.69 to 0.88 ) respectively, and DOR of 4.0 (95\% CI 2.4 to 6.9) [12], and in our study with enlarged sample size enrolling 19 trials of 2418 patients saw improved accuracy in diagnosis in patients with suspected sepsis.

However, the sample size of the trials we enrolled in this study was relatively small (in most of studies the participants not exceeding 100), which could lead to type II error with elevated false positive [33]. We thus conducted the sub-group analysis including studies enrolling more than 100 patients and found the pooled sensitivity of 0.71 (95\% CI 0.58 to 0.82 ), specificity of 0.69 (95\% CI 0.54 to 0.81 ) and DOR of 5.48 (2.10 to 14.27), which showed that the sensitivity, specificity and DOR all dropped compared with the overall results, suggesting more large-scale trials are needed for the evaluation of the diagnostic ability of sTREM-1.

The baseline characteristics of patients was complicated (post-operative, traumatic, mechanical ventilated), 


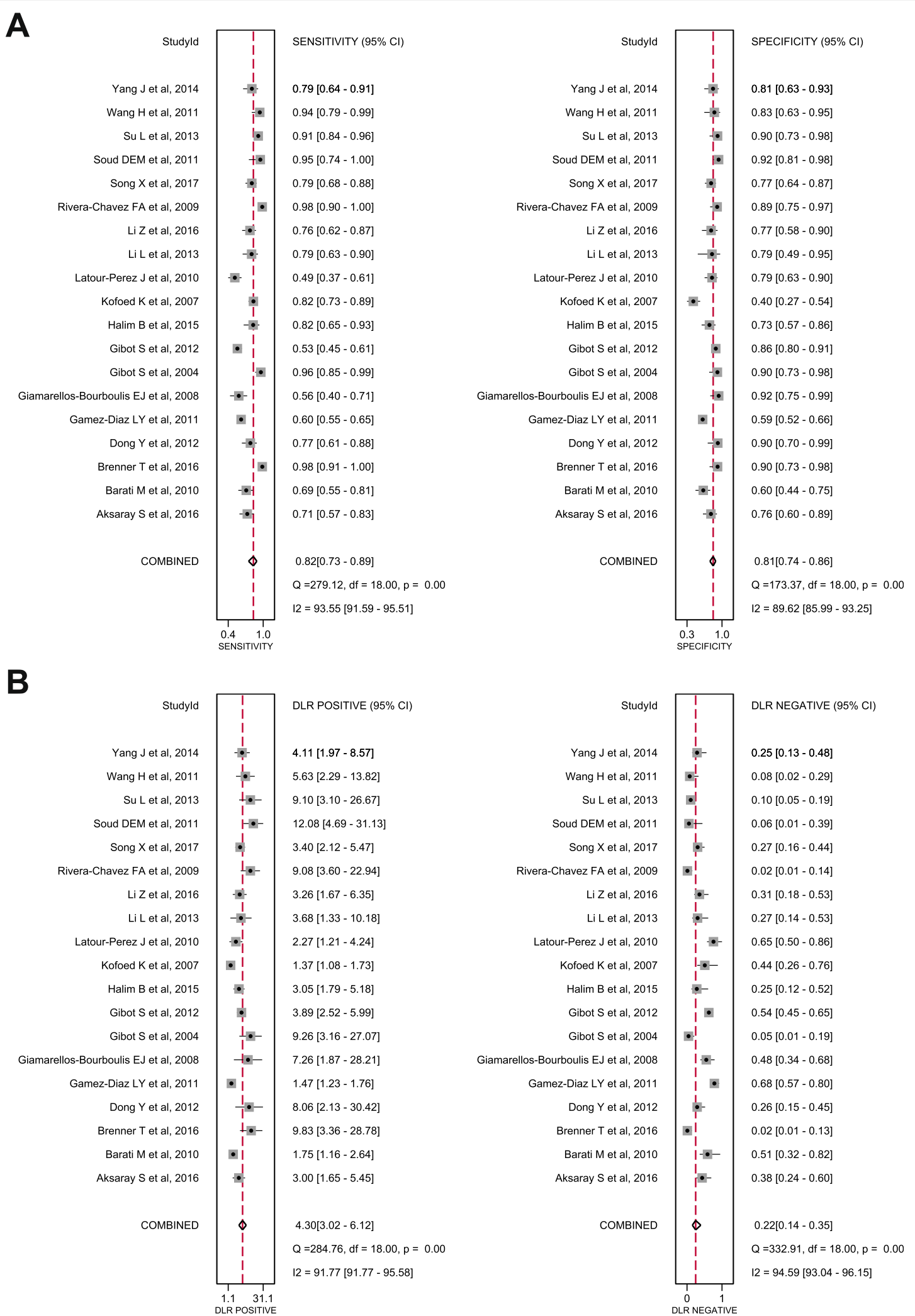

Fig. 3 Forest plots. a Forest plots showing the sensitivity $(0.82,95 \% \mathrm{Cl} 0.73-0.89)$ and specificity $(0.81,95 \% \mathrm{Cl} 0.74-0.86)$ of sTREM-1 in diagnosis in suspected sepsis; b Forest plots showing the positive likelihood ratio $(4.20,95 \% \mathrm{Cl} 3.02-6.12)$ and negative likelihood ratio $(0.22,95 \% \mathrm{Cl} 0.14-$ 0.35) of sTREM-1 in diagnosis in suspected sepsis 


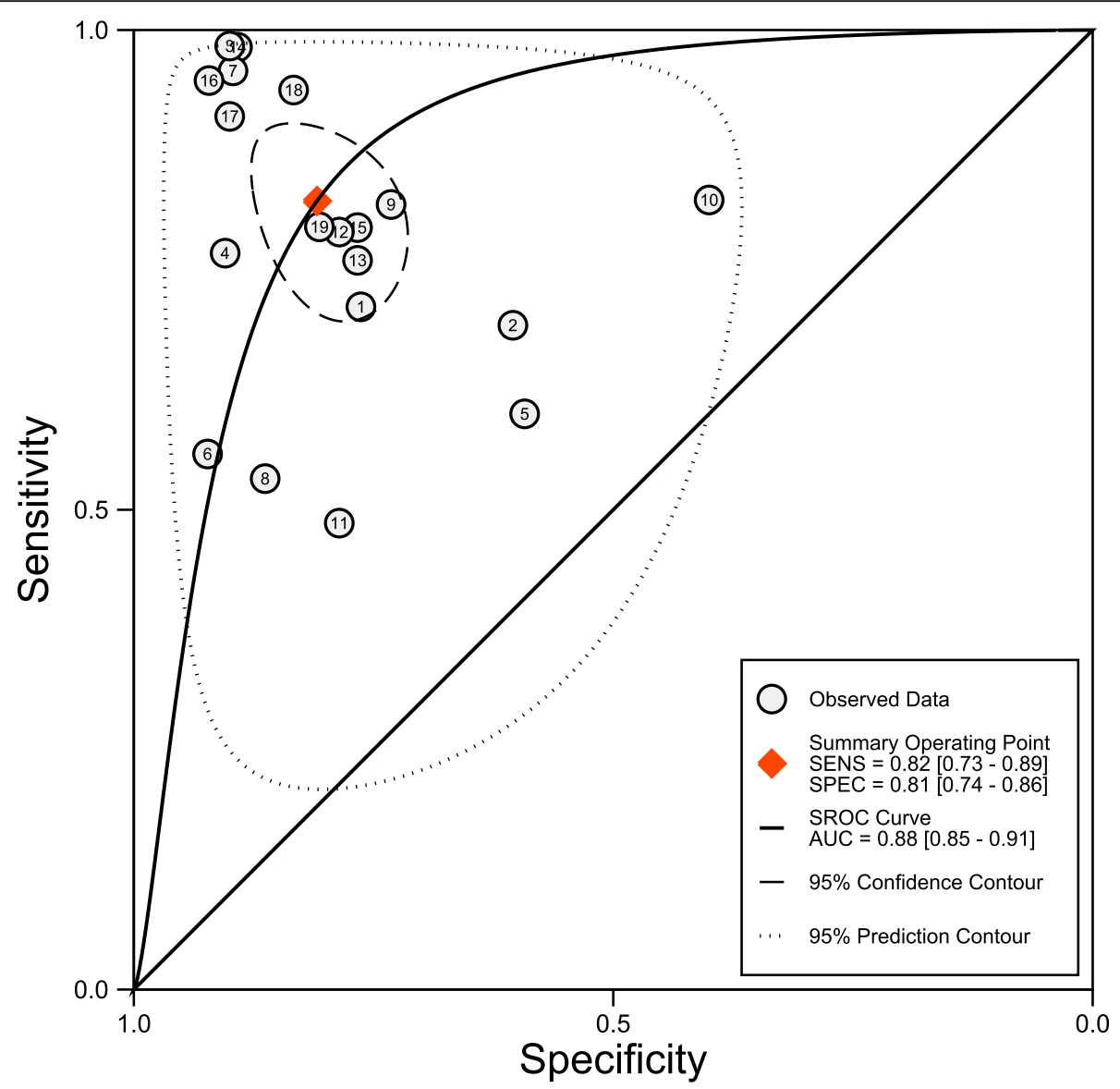

Fig. 4 Summary receiver operating curve

and heterogeneity in the patient selection might bring bias to the results; the severity of sepsis was different between studies with mortality ranged from $11 \%$ [27] to $54.4 \%$ [22], suggesting different pathogen virulence, inflammatory response and organ dysfunction, could probably influence the serum sTREM-1 level and its ability in discrimination of sepsis from SIRS. In some of the studies we included, not only patients with bacterial or fungal, but also with viral or parasite infection were recruited [23], and the heterogeneous composition of infection may also potentially bring bias to the results. The diagnosis of sepsis was relied on the comprehensive combination of clinical manifestation, laboratory and radiographic results, and the microbiological isolation or even in some occasions, the response to the empirical antibiotics therapy. We noticed that in the studies we included, the authors used inconsistent methods of determination of infection and in some studies only patients with positive pathogen isolation were included in the sepsis group [22, 27], all of which could potentially cause biases.

The level of serum sTREM-1 was also reported to be elevated in some non-infectious diseases in recent years, including chronic kidney disease on hemodialysis [34], chronic obstructive pulmonary disease [35], inflammatory bowel disease [36], atherosclerosis [37] and in malignant carcinomas like hepatocellular carcinoma [38] and non-small cell lung cancer [39], which could affect the specificity of sTREM-1 in sepsis diagnosis.

An ideal biomarker for sepsis should give information to the syndrome recognition, precise diagnosis and prognosis, and improve antibiotic stewardship [3], but unfortunately, no single biomarker could accomplish this task currently. In our study, sTREM-1 yielded a moderate diagnostic accuracy of sepsis. Currently, combination of biomarker for the diagnosis and prognostication of sepsis have been widely investigated as in the trial by Gibot et al [21], serum sTREM-1 were combined with PMN CD64 index and procalcitonin in diagnosis of sepsis.

\section{Limitations}

This study had several limitations. The sample size in most of the trials we included in this meta-analysis was relatively small (less than 100 participants). In some trials, the predictive ability of sTREM-1 in sepsis was also evaluated, however, was not included in our study. All 


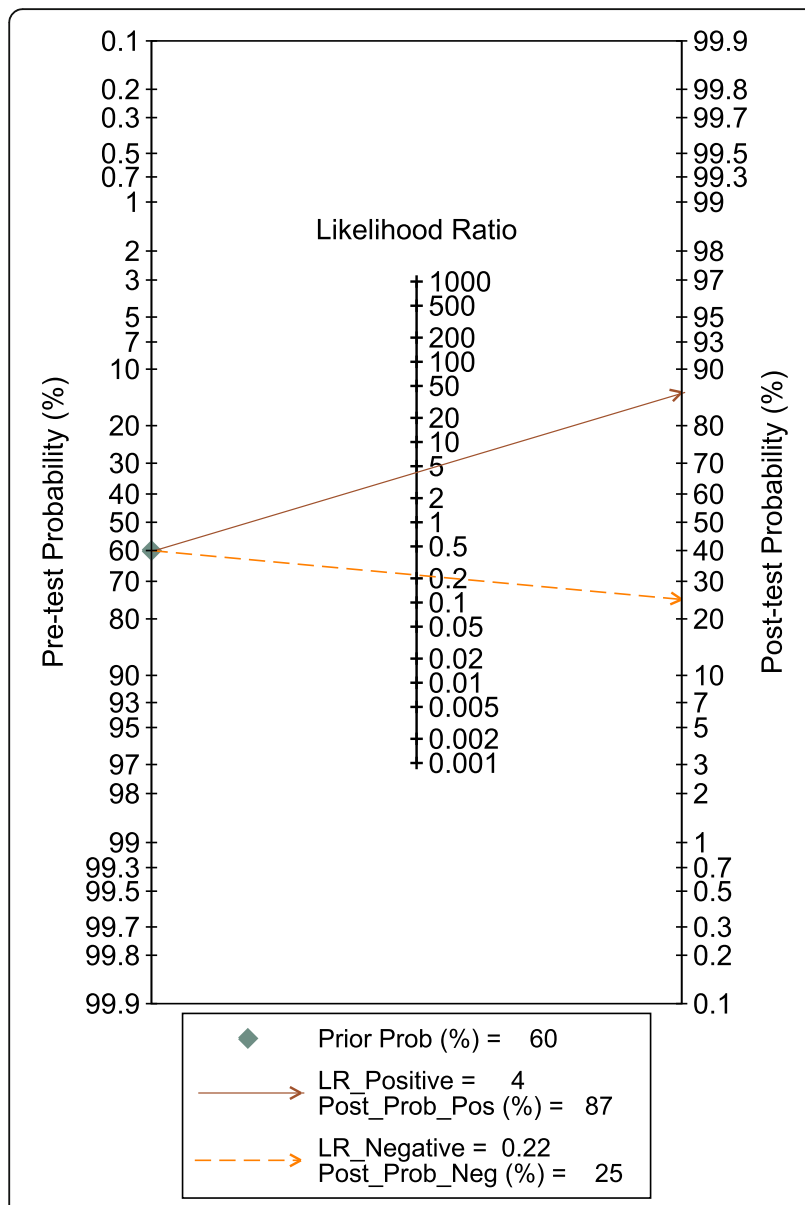

Fig. 5 Fagan's nomogram. Pre-test probability was set at $60 \%$, which yielded a post-test positive probability of $87 \%$ and negative of $26 \%$ the cut-off values in the trials included were not prespecified, but optimized by the sensitivity and specificity, so the cut-off value of sTREM-1 for sepsis diagnosis was not determined so far.

\section{Conclusions}

Serum sTREM-1 has a moderate accuracy in diagnosis in patients with suspected sepsis, however, the heterogeneity was high between studies. More large-scale studies are needed for validation the diagnostic value of sTREM-1 in suspected sepsis.

\section{Methods}

This manuscript was prepared following the guidelines of Preferred Reporting Items for Systemic Reviews and Meta-analyses (PRISMA) statement [40, 41] and Metaanalysis of Observational Studies in Epidemiology (MOOSE) statement [42]. This study has been registered in PROSPERO (CRD42018083695).

\section{Eligibility criteria}

We aimed to include all the clinical trials investigating serum STREM-1 as an early biomarker in the patients with suspected sepsis. The including criteria were as follows: (1) clinical trials of adult patients (>18 year-old) with suspected sepsis; (2) serum or plasma sTREM-1 protein expression was measured, if multiple measures were taken in the studies, only the earliest one was used; (3) a $2 \times 2$ contingency table with true positive (TP), false positive (FP), false negative (FN) and true negative (TN) could be constructed. As most of studies were conducted in prior of the upgrade to sepsis-3, sepsis was

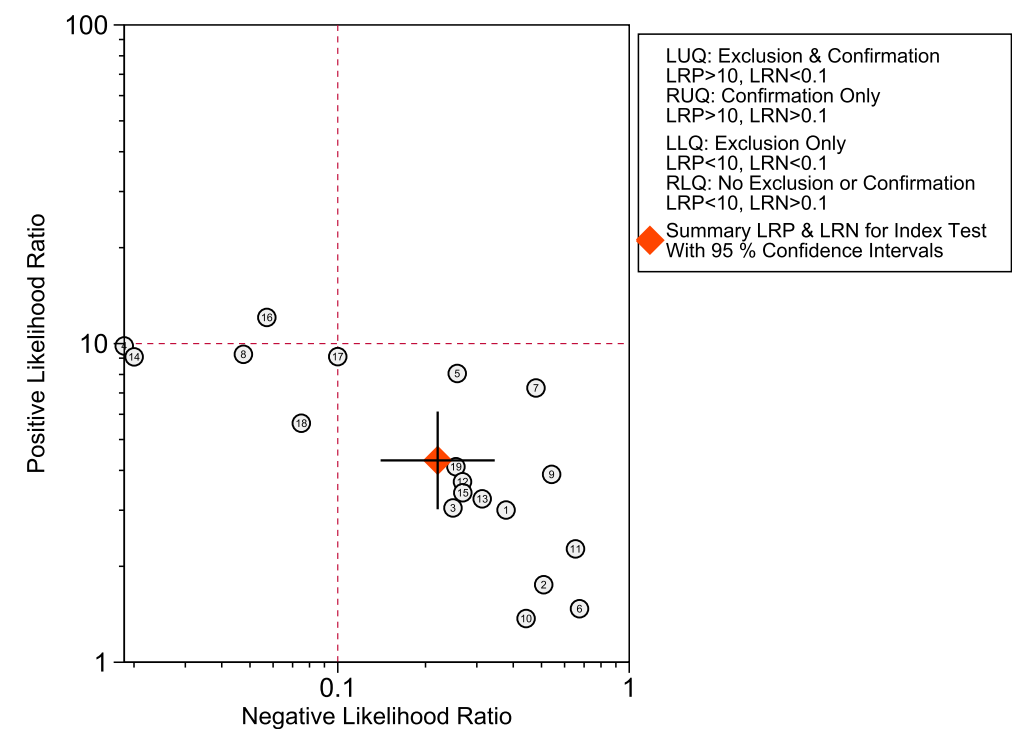

Fig. 6 Scattergram. The overall diagnostic accuracy of sTREM-1 was at the right lower quadrant with PLR $<10$ and NLR $>0.1$, which implying no exclusion or confirmation 


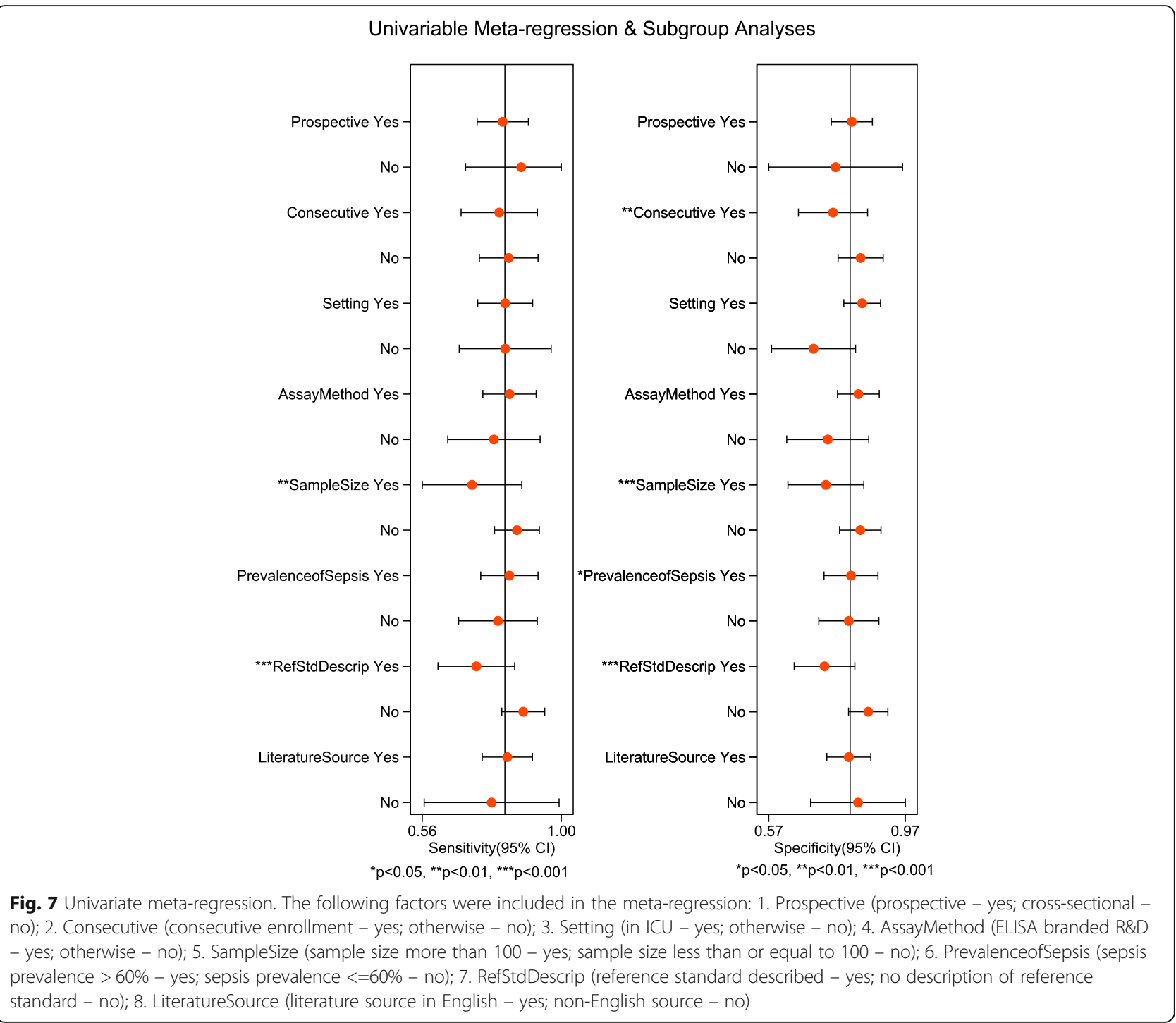

defined in accordance with the Surviving Sepsis Campaign Guidelines (2012) as the presence of infections together with the manifestation of systemic inflammatory response syndrome (SIRS) [43], and the determination of infection was remained to the researchers in individual studies.

\section{Information sources}

Two reviewers searched the electronic database including PubMed, EMBASE, Cochrane Central Register and Web of Science updated to June, 2018 separately, with no language restrictions. When relevant reviews or meta-analysis were reviewed, a backwards snowballing search was conducted for further studies.

\section{Search}

The following key words were used in our search strategy: "soluble triggering expressed receptor on myeloid cells 1", "triggering expressed receptor on myeloid cells 1", “sTREM-1", “TREM-1", "sepsis", "severe sepsis", "pyemia" and "septicemia". (Additional file 1).

\section{Study selection}

The titles and abstracts of the articles initially reviewed separately by two reviewers, and the full manuscripts were recruited if potentially relevant for further assessment. Disagreements were solved by consensus.

\section{Data extractions}

The following information was extracted for the analysis and assessment of the potential bias: (1) characteristics of study (design, settings, inclusion and exclusion criteria) and participants (sepsis prevalence, infection sites and microbiological features); (2) assay methods, cutoffs, sensitivity, specificity and the area under ROC curve (AuROC); (3) the procedures of diagnosis (may 


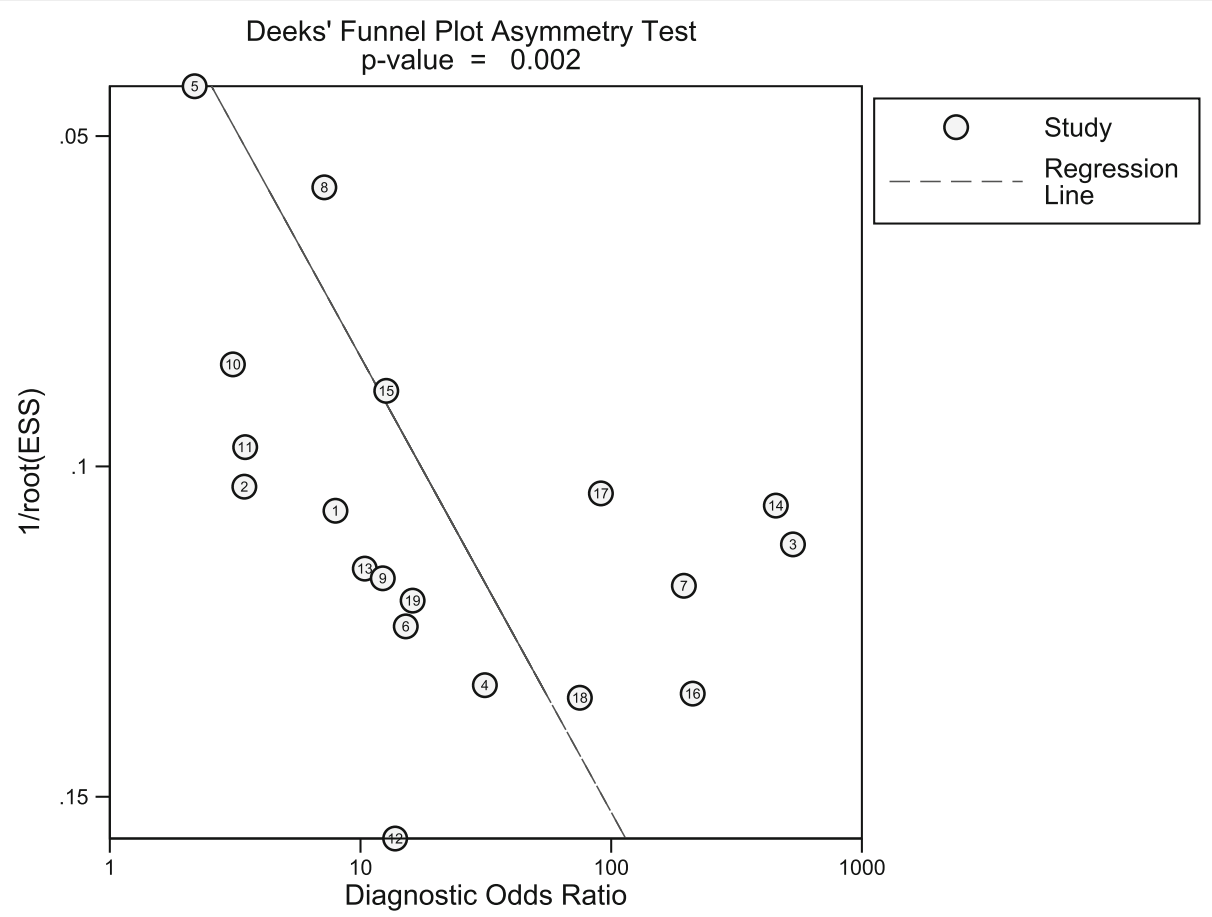

Fig. 8 Deeks' funnel plot. The $P$ value for the slope coefficient indicating significant asymmetry was 0.02 , which indicating a high likelihood of publication bias

including the clinical manifestation, laboratory and radiographic results, microbiological isolations); (4) the time point of sample obtain and diagnosis.

\section{Assessment of risk of Bias}

The internal validity and risk of bias of the included studies were evaluated by the Quality Assessment of Diagnostic Accuracy Studies 2 (QUADAS-2) tool [44], which consist of four domains including patient selection, index test, reference standard, and flow and timing, with the risk of bias assessed as "low", "unclear" and "high". Discrepancies were solved by consensus. (Additional file 2).

\section{Statistical analysis}

The number of patients classified in TP, FP, FN and TN were calculated from the prevalence of sepsis, sensitivity and specificity as provided in the studies. Data synthesis was performed within the bivariate mixed-effects regression framework to calculate average sensitivity and specificity, and also positive/negative likelihood ratio (PLR \& NLR) and diagnostic odds ratio (DOR, defined as PLR divided by NLR, which reflected the effectiveness of diagnosis), presented with 95\% confidence intervals (CI) [45].

The derived logit estimates of sensitivity, specificity and respective variance were used to construct a summary ROC curve, and the area under ROC (AuROC) was calculated for the global measure of the test performance, with $0.5>=$ AuROC $<=0.7$ as low, $0.7>=$ AuROC $<=0.9$ as moderate, and $0.9>=$ AuROC $<=1$ as high diagnostic accuracy [46]. The threshold effects were also visually assessed from the summary ROC, and the proportion of variance due to threshold effects was calculated as the squared correlation coefficient estimated from the between-study covariance parameter tested by rank correlation test.

Post-test probability was calculated using likelihood ratios based on Bayes' theorem and depicted visually with Fagan's nomograms [47]. The likelihood ratio scattergram was also plotted, with the definition of left upper quadrant of exclusion and confirmation, right upper quadrant of confirmation only, left lower quadrant of exclusion only and right lower quadrant of no exclusion or confirmation, respectively [48].

The factors that could potentially bring heterogeneity were extract and introduced in the univariate metaregression analysis, as we speculated from the designation of the studies, including study design (prospective or not, and consecutive or not), settings (in the ICU exclusively or not), assays (methods and kit brands), sample size ( $>100$ participants or not), prevalence of sepsis (according to the average prevalence of sepsis from the studies included), the description of the reference standard (described in detail or not) and literature source (published in English or not). Sub-analyses were subsequently conducted according to the results of the 
univariate meta-regression to investigate the diagnostic performances of sTREM-1 in sub-group population of patients.

The Deeks' funnel plot was used to evaluate the publication bias, with $P$ value $<0.10$ for the slope coefficient indicating significant asymmetry and a high likelihood of publication bias [49].

STATA (ver. 14, StataCorp LP, TX, USA) was used for the analyses, and midas command was used for the calculations. A two-tailed $P$ value $<0.05$ was considered statistical significance.

\section{Supplementary information}

Supplementary information accompanies this paper at https://doi.org/10. 1186/s12865-020-0332-x

Additional file 1. Full electronic search strategy on PubMed.

Additional file 2. Data extraction and study quality assessment protocol.

Additional file 3. Detailed characteristics and quality assessment of the included studies.

Additional file 4. Sub-group analysis according to the cut-off values.

Additional file 5: Figure S1. Sub-group analysis of studies conducted in ICU with patients consecutively recruited. The sub-group of 7 prospective trials conducted in the ICU, in which the patients with SIRS were consecutively recruited. A. Forest plots showing the sensitivity $(0.80,95 \%$ $\mathrm{Cl} 0.68-0.89)$ and specificity $(0.76,95 \% \mathrm{Cl} 0.64-0.84)$ of sTREM-1; B. Forest plots showing the positive diagnostic likelihood ratio (DLR positive) (3.30, 95\% Cl 1.98-5.50) and negative diagnostic likelihood ratio (DLR negative) (0.26, $95 \% \mathrm{Cl}$ 0.14-0.48) of sTREM-1.

Additional file 6: Figure S2. Sub-group analyses according to the meta-analysis results.

\section{Abbreviations}

AuROC: Area under ROC; Cl: Confidence interval; DOR: Diagnostic odds ratio; FN: False negative; FP: False positive; ICU: Intensive care unit; MOOSE: Metaanalysis of observational studies in epidemiology; NLR: Negative likelihood ratio; PLR: Positive likelihood ratio; PRISMA: Preferred reporting items for systemic reviews and meta-analyses; QUADAS-2: Quality assessment of diagnostic accuracy studies 2; ROC: Receiver operating characteristics: SIRS: Systemic inflammatory response syndrome; sTREM-1: soluble triggering receptor expressed on myeloid cells 1; TN: True negative; TP: True positive; TREM-1: Triggering receptor expressed on myeloid cells 1

\section{Acknowledgements}

Not applicable.

\section{Authors' contributions}

WC carried out the analysis and interpretation of data and submitting the manuscript. WC and FP participated in drafting, editing the manuscript, and elaborating the tables and figures. The articles were reviewed by two reviewers separately (WC and FP) in accordance to the inclusion criteria with discrepancies solved by consensus with a third reviewer (SSM). The quality assessment was conducted by two reviewers (JYX and SSM) with disagreements resolved by consensus including a third reviewer (FP). YY was responsible for conception, design and coordination of the study, and revision the manuscript for important intellectual contents. All the authors read and approve the final manuscript.

\section{Funding}

This study was supported by National Natural Science Foundation of China (Grant No. 81501705), National Natural Science Foundation of China (Grant No. 81671892), Natural Science Foundation of Jiangsu Province (Grant No. BK20161433) and Key Medical Subjects of Jiangsu Province (Grant No. ZDXKA2016025). The funding bodies have no roles in the design of the study; collection, analysis, and interpretation of data; and in writing the manuscript.

\section{Availability of data and materials}

The datasets used and/or analysed during the current study available from the corresponding author on reasonable request.

Ethics approval and consent to participate

Not applicable.

\section{Consent for publication}

Not applicable.

\section{Competing interests}

The authors declare that they have no competing interests.

Received: 13 August 2018 Accepted: 7 January 2020

Published online: 13 January 2020

\section{References}

1. Singer M, Deutschman CS, Seymour CW, et al. The third international consensus definitions for Sepsis and septic shock (Sepsis-3). JAMA. 2016; 315(8):801-10.

2. Rhodes A, Evans LE, Alhazzani W, et al. Surviving Sepsis campaign: international guidelines for Management of Sepsis and Septic Shock: 2016. Intensive Care Med. 2017:43(3):304-77.

3. Jensen JU, Bouadma L. Why biomarkers failed in sepsis. Intensive Care Med. 2016:42(12):2049-51.

4. Prucha M, Bellingan G, Zazula R. Sepsis biomarkers. Clin Chim Acta. 2015: 440:97-103.

5. Bouchon A, Dietrich J, Colonna M. Cutting edge: inflammatory responses can be triggered by TREM-1, a novel receptor expressed on neutrophils and monocytes. J Immunol. 2000;164(10):4991-5

6. Larsen FF, Petersen JA. Novel biomarkers for sepsis: a narrative review. Eur J Intern Med. 2017;45:46-50

7. Tessarz AS, Cerwenka A. The TREM-1/DAP12 pathway. Immunol Lett. 2008; 116(2):111-6.

8. Gomez-Pina V, Soares-Schanoski A, Rodriguez-Rojas A, et al. Metalloproteinases shed TREM-1 ectodomain from lipopolysaccharidestimulated human monocytes. J Immunol. 2007;179(6):4065-73.

9. Palazzo SJ, Simpson T, Schnapp LM. Triggering receptor expressed on myeloid cells type 1 as a potential therapeutic target in sepsis. Dimens Crit Care Nurs. 2012:31(1):1-6.

10. Cao C, Gu J, Zhang J. Soluble triggering receptor expressed on myeloid cell1 (sTREM-1): a potential biomarker for the diagnosis of infectious diseases. Front Med. 2017;11(2):169-77.

11. Jiyong J, Tiancha $\mathrm{H}$, Wei $\mathrm{C}$, et al. Diagnostic value of the soluble triggering receptor expressed on myeloid cells-1 in bacterial infection: a meta-analysis. Intensive Care Med. 2009:35(4):587-95.

12. Wu Y, Wang F, Fan X, et al. Accuracy of plasma sTREM-1 for sepsis diagnosis in systemic inflammatory patients: a systematic review and meta-analysis. Crit Care. 2012;16(6):R229.

13. Shi JX, Li JS, Hu R, et al. Diagnostic value of sTREM-1 in bronchoalveolar lavage fluid in ICU patients with bacterial lung infections: a bivariate metaanalysis. PLoS One. 2013;8(5):e65436.

14. Aksaray $S$, Alagoz $P$, Inan A, et al. Diagnostic value of sTREM-1 and procalcitonin levels in the early diagnosis of sepsis. North Clin Istanb. 2016; 3(3):175-82.

15. Barati M, Bashar FR, Shahrami $R$, et al. Soluble triggering receptor expressed on myeloid cells 1 and the diagnosis of sepsis. J Crit Care. 2010;25(2):362. e1-6.

16. Brenner T, Uhle F, Fleming T, et al. Soluble TREM-1 as a diagnostic and prognostic biomarker in patients with septic shock: an observational clinical study. Biomarkers. 2017;22(1):63-9.

17. Dong $Y$, Liang $X$. Soluble triggering receptor expressed on myeloid cells-1 were valuable in the diagnosis and assessment of severity of sepsis. Chin J Crit Care Med. 2012;32(2):115-9.

18. Gamez-Diaz LY, Enriquez LE, Matute JD, et al. Diagnostic accuracy of HMGB1, sTREM-1, and CD64 as markers of sepsis in patients recently admitted to the emergency department. Acad Emerg Med. 2011;18(8):807-15. 
19. Giamarellos-Bourboulis EJ, Mouktaroudi M, Tsaganos T, et al. Evidence for the participation of soluble triggering receptor expressed on myeloid cells-1 in the systemic inflammatory response syndrome after multiple trauma. J Trauma. 2008;65(6):1385-90.

20. Gibot S, Kolopp-Sarda MN, Bene MC, et al. Plasma level of a triggering receptor expressed on myeloid cells-1: its diagnostic accuracy in patients with suspected sepsis. Ann Intern Med. 2004;141(1):9-15.

21. Gibot S, Bene MC, Noel R, et al. Combination biomarkers to diagnose sepsis in the critically ill patient. Am J Respir Crit Care Med. 2012;186(1):65-71.

22. Halim B, Ozlem T, Melek C, et al. Diagnostic and prognostic value of procalcitonin and sTREM-1 levels in sepsis. Turk J Med Sci. 2015;45(3):57886.

23. Kofoed K, Andersen O, Kronborg G, et al. Use of plasma C-reactive protein, procalcitonin, neutrophils, macrophage migration inhibitory factor, soluble urokinase-type plasminogen activator receptor, and soluble triggering receptor expressed on myeloid cells-1 in combination to diagnose infections: a prospective study. Crit Care. 2007;11(2):R38.

24. Latour-Perez J, Alcala-Lopez A, Garcia-Garcia MA, et al. Diagnostic accuracy of sTREM-1 to identify infection in critically ill patients with systemic inflammatory response syndrome. Clin Biochem. 2010;43(9):720-4.

25. Li L, Zhu Z, Chen J, et al. Diagnostic value of soluble triggering receptor expressed on myeloid cells-1 in critically-ill, postoperative patients with suspected sepsis. Am J Med Sci. 2013;345(3):178-84.

26. Li Z, Zhao H, Zhao J, et al. The early diagnostic value and prognostic significance of serum presepsin (SCD14-ST) in patients with sepsis. Chin J Emerg Med. 2016;25(7):896-902.

27. Rivera-Chavez FA, Minei JP. Soluble triggering receptor expressed on myeloid cells-1 is an early marker of infection in the surgical intensive care unit. Surg Infect. 2009;10(5):435-9

28. Song $X$, Song $Y$, Zhang $X$, et al. Soluble triggering receptor expressed on myeloid Cells-1 as a novel marker for abdominal Sepsis. Surg Infect. 2017; 18(5):577-81.

29. Soud DEM, Amin OAl, Amin AAl. New era "soluble triggering receptor expressed on myeloid cells-I" as a marker for early detection of infection in trauma patients. Egypt J Anaesth. 2011;27(4):267-72.

30. Su L, Feng L, Song Q, et al. Diagnostic value of dynamics serum SCD163, sTREM-1, PCT, and CRP in differentiating sepsis, severity assessment, and prognostic prediction. Mediat Inflamm. 2013;2013:969875.

31. Wang HX, Chen B. Diagnostic role of soluble triggering receptor expressed on myeloid cell-1 in patients with sepsis. World J Emerg Med. 2011;2(3): 190-4.

32. Yang J, Huang $\mathrm{Y}, \mathrm{Wu} \mathrm{H}$. Clinical value of soluble triggering receptor expressed on myeloid cells-1(sTREM-1) in severe sepsis patients with pulmonary infiltrates. Chin J Crit Care Med. 2014;34(11):970-6.

33. Rucker G, Carpenter JR, Schwarzer G. Detecting and adjusting for smallstudy effects in meta-analysis. Biom J. 2011;53(2):351-68.

34. Essa ES, Elzorkany KM. sTREM-1 in patients with chronic kidney disease on hemodialysis. APMIS. 2015;123(11):969-74.

35. Phua J, Koay ES, Zhang D, et al. Soluble triggering receptor expressed on myeloid cells-1 in acute respiratory infections. Eur Respir J. 2006;28(4):695702.

36. Park JJ, Cheon JH, Kim BY, et al. Correlation of serum-soluble triggering receptor expressed on myeloid cells-1 with clinical disease activity in inflammatory bowel disease. Dig Dis Sci. 2009;54(7):1525-31.

37. Hermus L, Schuitemaker JH, Tio RA, et al. Novel serum biomarkers in carotid artery stenosis: useful to identify the vulnerable plaque? Clin Biochem. 2011; 44(16):1292-8

38. Duan M, Wang ZC, Wang XY, et al. TREM-1, an inflammatory modulator, is expressed in hepatocellular carcinoma cells and significantly promotes tumor progression. Ann Surg Oncol. 2015;22(9):3121-9.

39. Yuan Z, Mehta HJ, Mohammed K, et al. TREM-1 is induced in tumor associated macrophages by cyclo-oxygenase pathway in human non-small cell lung cancer. PLoS One. 2014;9(5):e94241.

40. Moher D, Liberati A, Tetzlaff J, et al. Preferred reporting items for systematic reviews and meta-analyses: the PRISMA statement. BMJ. 2009;339:b2535.

41. Liberati A, Altman DG, Tetzlaff J, et al. The PRISMA statement for reporting systematic reviews and meta-analyses of studies that evaluate healthcare interventions: explanation and elaboration. BMJ. 2009;339:b2700.

42. Stroup DF, Berlin JA, Morton SC, et al. Meta-analysis of observational studies in epidemiology: a proposal for reporting. Meta-analysis of observational studies in epidemiology (MOOSE) group. JAMA. 2000;283(15):2008-12.
43. Dellinger RP, Levy MM, Rhodes A, et al. Surviving Sepsis campaign: international guidelines for management of severe sepsis and septic shock, 2012. Intensive Care Med. 2013;39(2):165-228.

44. Whiting PF, Rutjes AW, Westwood ME, et al. QUADAS-2: a revised tool for the quality assessment of diagnostic accuracy studies. Ann Intern Med. 2011;155(8):529-36.

45. Reitsma JB, Glas AS, Rutjes AW, et al. Bivariate analysis of sensitivity and specificity produces informative summary measures in diagnostic reviews. J Clin Epidemiol. 2005;58(10):982-90.

46. Swets JA. Measuring the accuracy of diagnostic systems. Science. 1988; 240(4857):1285-93.

47. Fagan TJ. Letter: Nomogram for Bayes theorem. N Engl J Med. 1975;293(5): 257.

48. Stengel D, Bauwens K, Sehouli J, et al. A likelihood ratio approach to metaanalysis of diagnostic studies. J Med Screen. 2003;10(1):47-51.

49. Deeks JJ, Macaskill P, Irwig L. The performance of tests of publication bias and other sample size effects in systematic reviews of diagnostic test accuracy was assessed. J Clin Epidemiol. 2005;58(9):882-93.

\section{Publisher's Note}

Springer Nature remains neutral with regard to jurisdictional claims in published maps and institutional affiliations.
Ready to submit your research? Choose BMC and benefit from:

- fast, convenient online submission

- thorough peer review by experienced researchers in your field

- rapid publication on acceptance

- support for research data, including large and complex data types

- gold Open Access which fosters wider collaboration and increased citations

- maximum visibility for your research: over $100 \mathrm{M}$ website views per year

At BMC, research is always in progress.

Learn more biomedcentral.com/submissions 